\title{
Technological, functional and safety properties of lactobacilli isolates from soft wheat sourdough and their potential use as antimould cultures
}

\author{
Jatziri Mota-Gutierrez ${ }^{1} \cdot$ Irene Franciosa $^{1} \cdot$ Marianna Ruggirello $^{1} \cdot$ Paola Dolci $^{1}$ (D)
}

Received: 23 April 2021 / Accepted: 27 July 2021 / Published online: 7 August 2021

(c) The Author(s) 2021

\begin{abstract}
Bakery products are a common medium for fungal growth due to their high-water activity and nutrients availability. The application of lactic acid bacteria (LAB) isolated from wheat bran or other cereals has shown great potential in controlling the growth of spoilage fungi, guarantee quality and prolong the shelf life of bakery products. This study outlines the antifungal, technological, functional and safety properties of autochthonous LAB microbiota isolated from type 0 soft wheat sourdough fermentation. Antifungal activity of 77 LAB belonging to Lactiplantibacillus plantarum and Lacticaseibacillus casei species isolated from spontaneous sourdough fermentation was tested in vitro against 16 spoilage fungi. Our findings demonstrated that the antifungal activity, enzymatic and safety properties of LAB isolates vary strain-dependently. Four LAB isolates (Lp. plantarum A16, A25, B11, and B15) showed the best traits, in particular strong antifungal activity and good capabilities to produce exopolysaccharides from different carbon sources in vitro. Care should be taken when using Lp. plantarum $\mathrm{A} 310$ and B18 and Lc. casei A23, as starter cultures, since these isolates exhibited a multiple antibiotic-resistance. Here we showed the promising potential of different LAB isolates as bio-preservative agents and to provide new insights regarding their prospective use as starter cultures to guarantee safety and palatability.
\end{abstract}

Paola Dolci

paola.dolci@unito.it

1 Department of Agricultural, Forest and Food

Sciences, University of Turin, Largo Paolo Braccini 2,

10095 Grugliasco, Turin, Italy 


\section{Graphic abstract}

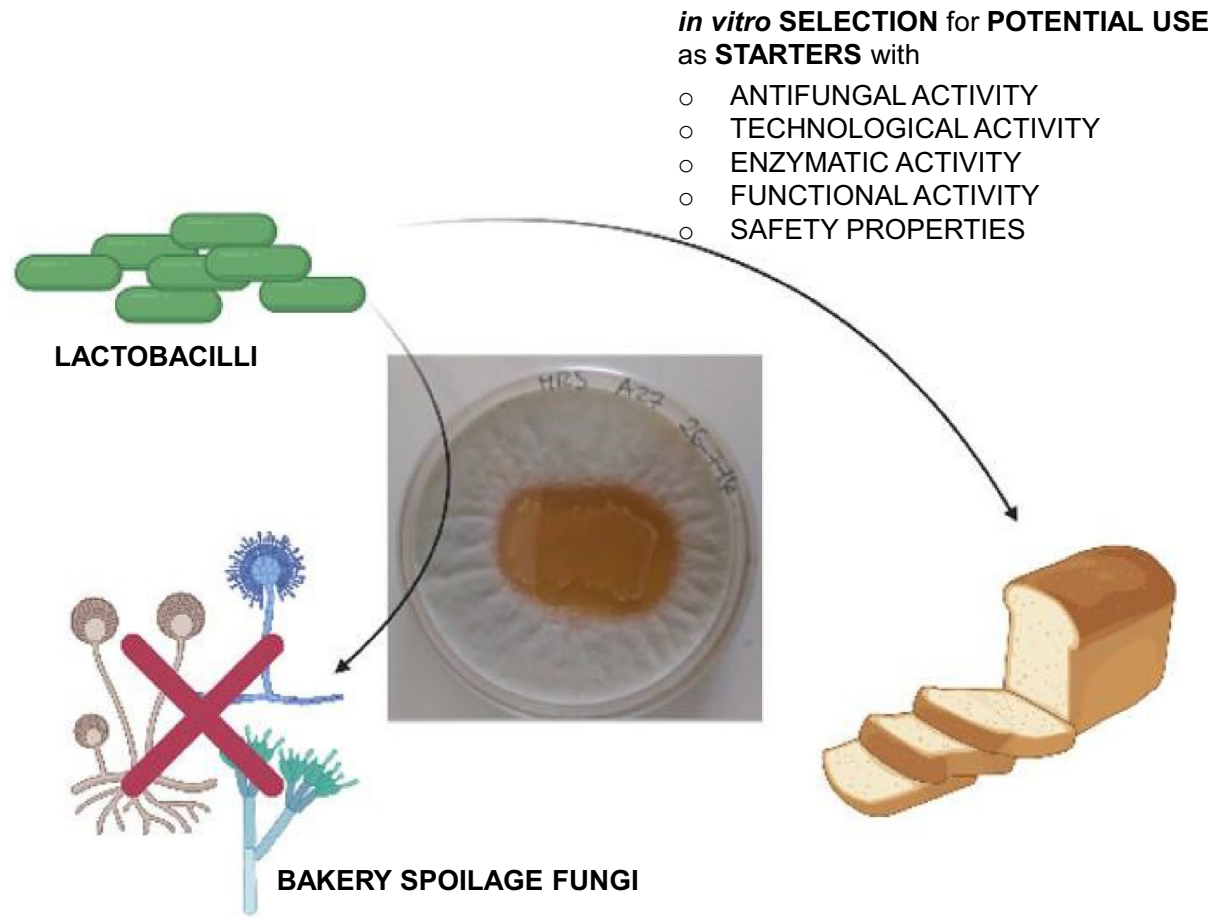

Keywords Fungi $\cdot$ Lactic acid bacteria $\cdot$ Lactiplantibacillus plantarum $\cdot$ Lacticaseibacillus casei $\cdot$ Wheat $\cdot$ Antifungal activity

\section{Introduction}

Cereals and cereal products, such as bread, are considered a staple food. The high content in carbohydrates, dietary fiber and protein sources of bread, makes this cereal product one of the main dietary sources for human nutrition but also a desirable substrate for mold contaminants. The contamination sources of bakery products are mostly environmentally based, coming from the air, handling, processing equipment and storage, as well as from the flour itself (Rosenkvist and Hansen 1995; Reale et al. 2013; Garcia et al. 2019). Aspergillus, Penicillium, Fusarium, Rhizopus and Mucor are the most common mold genera associated with bakery product spoilage (Legan 1993; Garcia et al. 2019). Fungi are responsible for off-flavors and production of mycotoxins which belong to the most toxic contaminants occurring in a wide range of food commodities (Bennet and Wallsgrove 1994).

Research in the field of characterization and selection of natural bio-protective agents that also guarantees quality and ensure safety is becoming increasingly important to promote the use of eco-friendly alternatives instead of using chemical additives in the food industry. The use of Lactiplantibacillus plantarum strains as natural bio-protective agents has emerged as a sustainable alternative for food conservation.
The antifungal, technological, safety and nutritional properties of Lp. plantarum strains such as broad-range activity towards spoilage fungi, high stability at various $\mathrm{pH}$ and temperatures, and production of bacteriocins have been extensively studied (De Angelis et al. 2003; Gupta and Srivastava 2014; Manini et al. 2016; Ruiz Rodríguez et al. 2016; Arena et al. 2019). Besides Lp. plantarum, Levilactobacillus brevis, Limosilactobacillus reuteri, Furfurilactobacillus rossiae strains isolated from sourdough have been demonstrated to control mold growth and the production of aflatoxin, as well as showing technological and enzymatic activities (Garofalo et al. 2012; Manini et al. 2016; Sadeghi et al. 2019a, b).

The technological and functional characteristics of LAB strains used in the food chain contribute to the sensory quality of food products (De Vuyst et al. 2010; Viana de Souza and Silva Dias 2017). In particular, LAB are able to enrich dough, producing exopolysaccharides (EPS), enzymes, organic acids and antimicrobial compounds (Torrieri et al. 2014). In this regards, the characterization of LAB strains isolated mainly from wheat germ, wheat bran, and hard wheat has been extensively investigated (Minervini et al. 2010; Garofalo et al. 2012; Manini et al. 2016; Sadeghi et al. 2019a, b; Fekri et al. 2020). Whereas somehow the characterization of LAB strains and the identification of the 
microbial composition of soft wheat is rarely investigated (Nionelli et al. 2014; Taccari et al. 2016). Soft wheat is per capita the main grain consumed, followed by maize and rye (Statistik Austria, 2021). The high consumption of wheat is linked to the vast number of products containing wheat as the main ingredient such as bread, pasta, other bakery products and is used for thickening gravy and sauces. In this study, LAB isolates were chosen from soft wheat sourdough for its nutritional characteristics, such as less starch and gluten content compared with bran wheat (USDA, 2020). Despite many studies, it is still unclear to what extent the sourdough microbiota is affected and selected by the type of flour used. For sure, cereal fermentation processes depend on specific determinants, and the type of flour is one of the most important (Nionelli et al. 2014; Taccari et al. 2016).

The unique characteristics of the different types of sourdough influence also the microbial ecosystem (Gänzle and Zheng 2019). In spontaneous sourdough fermentation, LAB such as Lp. plantarum, Lacticaseibacillus casei, Fl. rossiae, and pediococci, dominate the microbial ecosystem (Gänzle and Zheng 2019; Minervini et al. 2010). In bakery industry, different LAB species have been used as a starter culture or co-culture to improve the sensorial attributes and direct microbial development (Salmenkallio-Marttila et al., 2001). Although LAB have been applied for decades in food preservation, leading to their classification as Generally Recognized As Safe (GRAS), there is growing evidence that $L p$. plantarum, Ll. brevis and Latilactobacillus curvatus isolated from sourdough can be resistant to antibiotics such as clindamycin (Manini et al. 2016). In this research, we studied autochthonous LAB strains previously isolated from spontaneous fermented sourdough-like soft wheat flour for their antifungal activity to explore their bioprotective potential. Thus, the best performing LAB isolates were characterized for their technological, functional and safety properties to provide new insights towards the potential use of new LAB isolates as starter cultures in food applications, such as wheat fermentation and sourdough bread, without compromising food safety.

\section{Materials and methods}

\section{LAB isolate selection based on antifungal activity}

In total, seventy-seven LAB cultures, identified as $L p$. plantarum $(n=68)$ and Lc. casei $(n=9)$ by species-specific PCR (Berthier and Ehrlich, 1998) and $16 \mathrm{~S}$ rRNA gene sequencing (Teymoortash et al. 2002), respectively, were revived from glycerol stock stored at $-80^{\circ} \mathrm{C}$ at DISAFA Collection (Department of Agricultural, Forest, and Food Sciences, University of Turin), in De Man, Rogosa and Sharpe (MRS, Oxoid, Milan, Italy) broth twice and incubated at $30{ }^{\circ} \mathrm{C}$ for
$24 \mathrm{~h}$. LAB strains had been previously isolated from soft wheat sourdoughs produced in both artisanal and industrial bakeries located in northwest Italy. Precisely, LAB strains coded with "A" came from a company (located in Piedmont region) producing the traditional Italian products Colomba and Panettone; LAB strains coded with "B" from an artisanal bakery (located in Piedmont region) mainly producing bread; and with "C" from an artisanal bakery (located in Aosta Valley region) producing Panettone. Sixteen mold strains were revived in Malt Extract Agar (Oxoid) at $27^{\circ} \mathrm{C}$ for 5 days. They had been previously isolated from bakery products (moldy bread and Panettone) and stored at DISAFA Collection or purchased from the Mycotheca Universitatis Taurinenesis (MUT) (Department of Life Sciences and Systems Biology, University of Turin) and the Federal Research Centre for Nutrition and Foods (BFE) (Karlsruhe, Germany) (Table 1).

The seventy-seven LAB cultures were preliminarily screened for their antifungal activity against three fungal strains selected in this study as main targets (Aspergillus fumigatus, Aspergillus niger and Penicillium expansum). Twenty LAB isolates (Lp. plantarum $=17$ and Lc. case $i=3$ ) were preselected for their stronger antifungal activity against the fungal target cultures and further tested against the rest of the molds $(n=13)$.

The antifungal activity of LAB isolates was determined using the overlay method described by Magnusson and Schnürer with some modifications (Magnusson and Schnürer 2001). From Malt Extract Agar plates, the conidia were scraped and harvested from mycelium surface with Ringer solution (Oxoid) and counted by means of Burker chamber. $\mathrm{LAB}$ isolates were inoculated in two 2-cm-long lines on MRS agar plates and incubated at $37^{\circ} \mathrm{C}$ for $24 \mathrm{~h}$. The plates were then overlaid with $10 \mathrm{~mL}$ of Malt Extract Soft Agar (Biolife, Milan, Italy) inoculated with $10^{4}$ conidia/mL of the fungal suspension. The overlaid plates were incubated at $25^{\circ} \mathrm{C}$ for 5 days. Plates were then examined for clear zones of inhibition around the LAB streaks and scored as - (no growth suppression), + (medium growth suppression) and ++ (total growth suppression). Inhibition tests were done in triplicates.

\section{Growth and acidification rate of selected LAB isolates}

The microbial counts, $\mathrm{pH}$ and Total Titratable Acidity (TTA) were analyzed to determine the fermentation capacity of the twenty pre-selected LAB isolates following Manini et al. (2016) protocols with some modifications. An overnight culture $(1 \% \mathrm{v} / \mathrm{v})$ of each LAB isolate was individually inoculated into a sample $(100 \mathrm{~g})$ of type "0" soft wheat flour (15\% $\mathrm{w} / \mathrm{v}$ of flour and $85 \%$ of water) and incubated for $7 \mathrm{~h}$ at $30^{\circ} \mathrm{C}$ on a horizontal shaker Promax 2020 (Heidolph, Schwabach, 


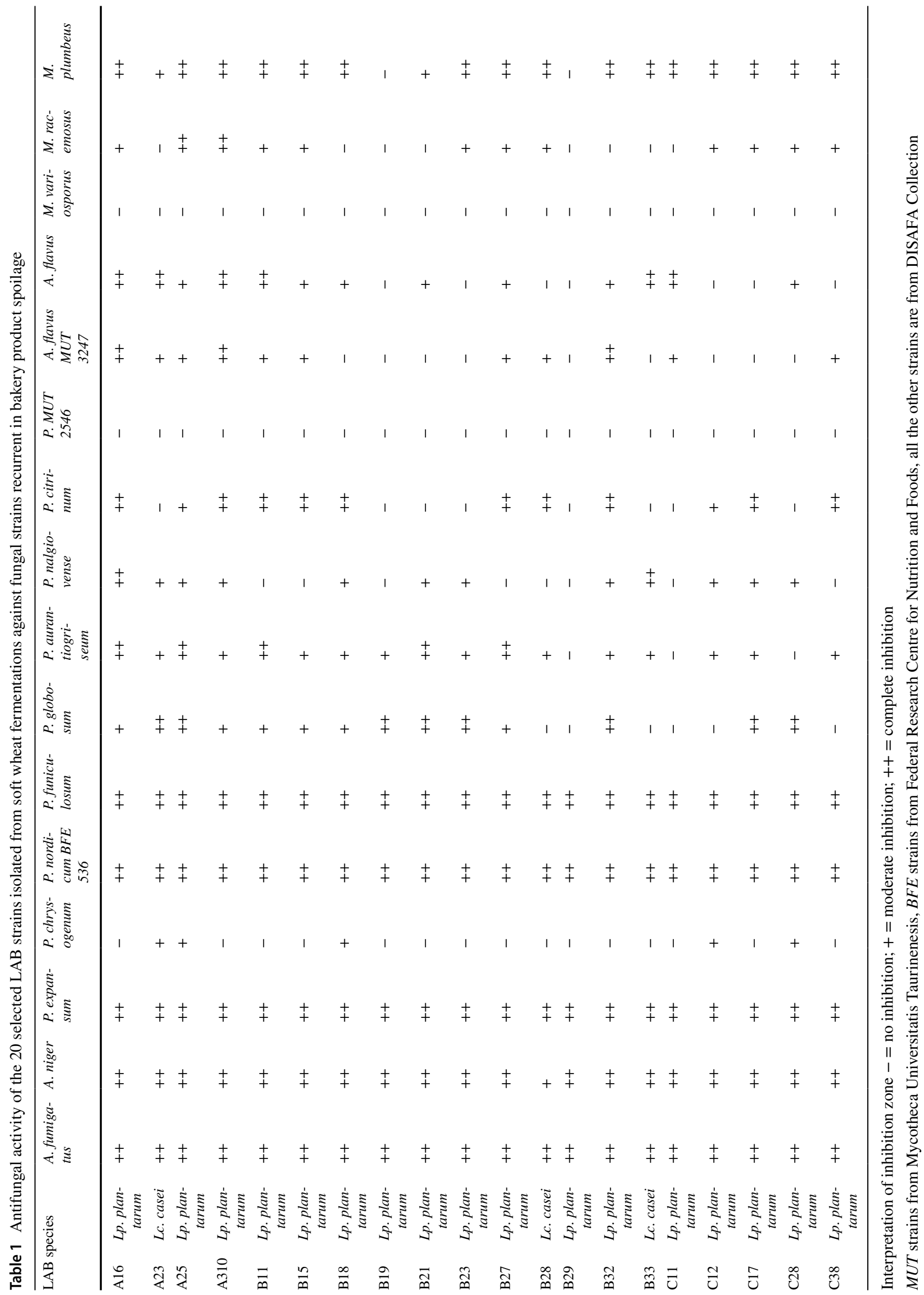


Germany). Classical microbiological analysis was performed immediately after LAB inoculum and after $7 \mathrm{~h}$ of fermentation. MRS agar was used for counting lactobacilli incubated at $30{ }^{\circ} \mathrm{C}$ for $48-72 \mathrm{~h}$. The dynamics of $\mathrm{pH}$ and TTA were measured after inoculum and during the fermentation at 4 and $7 \mathrm{~h}$. The kinetics of acidification by LAB isolates were measured from the suspension of $10 \mathrm{~g}$ sample of sourdough in $100 \mathrm{~mL}$ of deionized water. For the assessment of TTA, this suspension was titrated with $0.1 \mathrm{M} \mathrm{NaOH}$ to a final $\mathrm{pH}$ of 8.5. Results of TTA were expressed as milliliters of $0.1 \mathrm{M}$ $\mathrm{NaOH}$ needed to achieve the final $\mathrm{pH}$ of 8.5. All samples were analyzed in triplicate.

\section{Technological, functional and safety properties of selected LAB isolates}

\section{Exopolysaccharide (EPS) production}

Overnight LAB cultures were plated on different MRS agar media with glucose, sucrose, maltose, lactose, and starch as the only carbon sources. Plates were incubated at $30{ }^{\circ} \mathrm{C}$ for $48 \mathrm{~h}$. Triplicate plates of each LAB were scored for mucoid properties (scale of $++=$ excess EPS,$+=$ moderate EPS and $-=$ no visible mucoid). Colonies were scored as positive if strings were detected when the colony was touched once with an inoculating loop (Manini et al. 2016).

\section{Xylanase activity}

To test xylanase producing microorganisms at different $\mathrm{pH}$, two different agar media were prepared by adding $0.1 \%$ (w/v) of the dyed substrate (Remazolbrilliant Blue R plus Azo-Xylan - birchwood) to a sodium phosphate buffer, 100 $\mathrm{mM}, \mathrm{pH} 6$ and to a sodium acetate buffer, $100 \mathrm{mM}, \mathrm{pH} 4.5$ (Manini et al. 2016). Triplicate plates were inoculated with $2 \mu \mathrm{L}$ of the overnight LAB cultures and incubated at $30^{\circ} \mathrm{C}$ for $48 \mathrm{~h}$. The xylanase activity was determined based on the diameter of the clearing zone of inhibition around the LAB streaks.

\section{Amylolytic activity}

The starch degradation by spot inoculation of LAB isolates was evaluated in triplicates, as described by Rodriguez et al. (2016) with some modifications. LAB isolates $(2 \mu \mathrm{L})$ were streaked on plates containing MRS-starch medium in which glucose was replaced by starch (1\%). Inoculated plates were incubated at $30{ }^{\circ} \mathrm{C}$ for $48 \mathrm{~h}$ and then stored at $4{ }^{\circ} \mathrm{C}$ for $24 \mathrm{~h}$. After $72 \mathrm{~h}$ of incubation, plates were flooded with iodine solution (4\%). Amylase production was indicated by a clear zone around the colonies, while the rest of the plate stained blue-black. Amylolactobacillus amylophilus 20,533 was used as a positive control strain. The amylolytic activity was determined based on the diameter of the clearing zone of inhibition around the LAB streaks.

\section{Phytase activity}

LAB isolates preliminary inoculated in MRS broth and incubated at $37{ }^{\circ} \mathrm{C}$ for $24 \mathrm{~h}$ were then growth at $30{ }^{\circ} \mathrm{C}$ for $48 \mathrm{~h}$ in modified Chalmers broth with $1 \%$ neutral red and $1 \%$ calcium phytate (Manini et al. 2016). Phytase activity was then determined based on the diameter of the clearing zone around the holes in the modified Chalmers agar plates. To eliminate false positive results, caused by microbial acid production, Chalmer agar plates were flooded twice with $2 \%(\mathrm{w} / \mathrm{v}$ ) aqueous cobalt chloride solution. After $20 \mathrm{~min}$, the solution was removed and phytase activity evaluated (Anastasio et al. 2010).

\section{Proteolytic activity}

Proteolysis of LAB cultures was assayed against gelatin or casein on agar plates prepared as follows: $2 \%$ sucrose, $0.5 \%$ yeast extract, $2 \%$ peptone, $1.5 \%$ agar autoclaved and supplemented with $1 \%$ gelatin or casein, and incubated at $37^{\circ} \mathrm{C}$ for $48 \mathrm{~h}$. Extracellular protease activity was detected after staining agar plates with Coomassie blue $(0.25 \%$, w/v) for $1 \mathrm{~h}$ in methanolacetic acid-water 5:1:4 (v/v/v) and destaining with methanolacetic acid-water. Areas of enzymatic activity were scored as grade -, when no visible halo was present; + , when visible proteolysis was limited to $1-2 \mathrm{~mm}$ around the colony; and ++ , when the zone of proteolysis was more than $2 \mathrm{~mm}$ from the colony.

\section{Antibiotic resistance}

The minimum inhibitory concentration (MIC) of eight antibiotics (ampicillin-Amp, gentamicin-Gen, kanamycin-Kan, streptomycin-Str, erythromycin-Ery, clindamycin-Cli, tetracycline-Tet, chloramphenicol-Cho) was determined by the microdilution method as reported by the ISO 10,932:2010 standard method (ISO 10,932/IDF 223, 2010). Seven LAB isolates, selected according to their strong antifungal activity, EPS production and enzymatic activity, were classified as resistant/sensitive according to the cut-off values defined according to the FEEDAP Panel (EFSA-FEEDAP2012).

\section{Statistical analysis}

The results are presented as mean values \pm standard deviation of triplicate measurements. Statistical analyses were performed using generalized linear mixed-effects model for non-distributed data set. Mixed models were chosen for their ability to capture both fixed (LAB isolates) and random effects (number of replicates). When no dispersion 
of the data distribution was observed (EPS production, proteolytic activity, antifungal activity) any statistical test was used. The $P$-values were adjusted using Bonferroni's method; when the linear mixed model revealed significant differences $(P<0.05)$ the Duncan honestly significant difference (HSD) test was applied. Mixed models were built and evaluated following Crawley (Crawley 2007), using $\mathrm{R}$ version 3.3.2.

\section{Results}

\section{LAB isolates showed different inhibitory activity against fungal species}

The antifungal assays showed that all the 20 selected LAB isolates were able to inhibit A. fumigatus, A. niger, P. expansum, Penicillium nordicum, and Penicillium funiculosum. Overall, LAB isolates were capable of inhibiting the growth of 5 up to 13 fungal species (Table 1). Lp. plantarum A16, followed by A310 demonstrated strong antifungal activity against 11 and 10 fungal species, respectively, whereas Lp. plantarum B29 showed strong antifungal activity against only 5 fungal species. In contrast, none of LAB isolates were able to inhibit the growth of Mucus variosporus and Penicillium sp. MUT 2546 (Table 1).

\section{Significant differences between growth and $\mathrm{pH}$ rate of LAB isolates}

The growth, in sourdough medium, of all the tested LAB isolates increased substantially after $7 \mathrm{~h}$ of incubation (average increase approximately $1.47 \log \mathrm{CFU} / \mathrm{g}$, Table 2). A significant difference in the growth rate, $\mathrm{pH}$ values, and TTA was observed among LAB isolates (Table 2). In detail, significantly higher loads of $L p$. plantarum B27 were observed at the beginning and the end of the fermentation (9.20 and $11.06 \log$ CFU/g, respectively), while Lp. plantarum A310 and $\mathrm{B} 11$ showed the lowest load at the end of the fermentation (8.26 and $8.38 \mathrm{log}$ CFU/g, respectively, $P<0.05$ Table 2). Concerning $\mathrm{pH}$ value changes, we observed significantly higher $\mathrm{pH}$ values for $L c$. casei A23 (5.19) and lowest values for Lp. plantarum A16, A25, B29, B32, C11 (4.15, $4.15,4.15,4.19$ and 4.14 , respectively, $P<0.05$, Table 2$)$ at the end of the fermentation. In parallel, significantly higher total acidity was observed for Lp. plantarum B29, C11 and C12 (2.20, 2.38 and 2.18, respectively), while significantly lower acidity was reported for $L p$. plantarum B27 and $L c$. casei A23 (1.08 and 0.65, respectively, $P<0.05$, Table 2) at the end of the fermentation.

\section{Effect of technological, functional and safety properties of LAB isolates}

\section{LAB isolates showed different abilities to biosynthesize EPS}

Overall, most of the LAB strains were able to grow on maltose, glucose, sucrose, and lactose $(11,11,10$ and $9 \mathrm{LAB}$ isolates, respectively, Table 3). Lp. plantarum A16 and B11 produced EPS using different carbon sources as shown in Table 3. Lp. plantarum A16, B11, B15, B27, B32, and C28 strains were only able to produce EPS while growing on starch (Table 3). Interestingly, 2 out of the 20 LAB strains did not produce EPS (Lp. plantarum B19 and B28, Table 3).

\section{Significant differences in the xylanase activity of LAB isolates}

Thirteen out of $20 \mathrm{LAB}$ isolates showed an activation zone diameter ranging between 1.00 and $5.33 \mathrm{~mm}$ in plate assay with sodium phosphate buffer (Table 3 ). Interestingly, when the plates contained sodium acetate buffer, a significant difference between the xylanase activity of LAB isolates was observed (Table 3, $P<0.05$ ). In detail, significantly higher xylanase activity was detected in Lp. plantarum B11 and B23, reaching an activity zone diameter of $7.66 \mathrm{~mm}$, while the lowest activity was observed in Lp. plantarum A16, B27, B32, B33, C11, in which the activity zone diameter reached $6.33 \mathrm{~mm}(P<0.05$, Table 3$)$.

\section{LAB isolates exhibited a significant different amylolytic activity}

A significantly higher amylolytic activity was detected in Lp. plantarum B11 and B15, reaching an activity zone diameter of $4 \mathrm{~mm}$, while the lowest activity was observed in $L p$. plantarum $\mathrm{C} 28$, in which the activity zone diameter reached $1 \mathrm{~mm}(P<0.05$, Table 3$)$. Overall, the amylolytic activity was displayed by all LAB isolates, except for Lc. casei B33 (Table 3).

\section{LAB phytases are apparently not affected by LAB diversity}

The ability to hydrolase hexacalcium phytate was present among all the LAB isolates tested. Overall, sixteen out of $20 \mathrm{LAB}$ isolates exhibited a high inhibiting zone diameter, reaching 2.0-2.5 $\mathrm{mm}$ (Table 3 ).

\section{Casein agar plates induced a higher number of proteolytic reactions from $L A B$ isolates}

Only two LAB isolates were able to degrade gelatin in plate assays as observed by the formation of a clear halo after incubation on a medium containing gelatin (Lp. plantarum 
Table $2 \mathrm{LAB}$ viable cell-counts ( $\log \mathrm{CFU} / \mathrm{g}), \mathrm{pH}$ and total titratable acidity variations in $7 \mathrm{~h}$ sourdough fermentation

\begin{tabular}{|c|c|c|c|c|c|c|c|c|c|c|c|c|c|c|c|}
\hline \multicolumn{3}{|l|}{$\mathrm{LAB}$ isolate } & \multicolumn{4}{|l|}{$0 \mathrm{~h}$} & \multicolumn{4}{|l|}{$4 \mathrm{~h}$} & \multicolumn{4}{|l|}{$7 \mathrm{~h}$} & \multirow[t]{2}{*}{ Dynamics } \\
\hline Lp. plantarum & A16 & & & & & & & & & & & & & & \\
\hline & & $\mathrm{pH}$ & 6.60 & \pm & 0.07 & $a b c$ & 5.63 & \pm & 0.06 & $\mathrm{c}$ & 4.15 & \pm & 0.01 & $\mathrm{c}$ & 2.45 \\
\hline & & TTA & 0.35 & \pm & 0.07 & $a b c$ & 0.63 & \pm & 0.04 & & 1.93 & \pm & 0.04 & $a b c$ & -1.58 \\
\hline & & $\log \mathrm{CFU} / \mathrm{g}$ & 7.99 & \pm & 0.01 & $\mathrm{bc}$ & nd & & & & 9.72 & \pm & 0.02 & $a b$ & 1.73 \\
\hline \multirow[t]{4}{*}{ Lc. casei } & $\mathbf{A 2 3}$ & & & & & & & & & & & & & & \\
\hline & & $\mathrm{pH}$ & 6.78 & \pm & 0.03 & $a b$ & 6.21 & \pm & 0.09 & $a b$ & 5.19 & \pm & 0.04 & $\mathrm{a}$ & 1.59 \\
\hline & & TTA & 0.20 & \pm & 0.00 & bcde & 0.38 & \pm & 0.04 & & 0.65 & \pm & 0.42 & $\mathrm{c}$ & -0.22 \\
\hline & & $\log \mathrm{CFU} / \mathrm{g}$ & 7.62 & \pm & 0.17 & $\mathrm{c}$ & nd & & & & 9.34 & \pm & 0.15 & $\mathrm{~b}$ & 1.72 \\
\hline \multirow[t]{4}{*}{ Lp. plantarum } & A25 & & & & & & & & & & & & & & \\
\hline & & $\mathrm{pH}$ & 6.63 & \pm & 0.00 & $a b c$ & 5.45 & \pm & 0.15 & $\mathrm{c}$ & 4.15 & \pm & 0.01 & $\mathrm{c}$ & 2.48 \\
\hline & & TTA & 0.30 & \pm & 0.00 & abcd & 0.65 & \pm & 0.07 & & 1.70 & \pm & 0.14 & $a b c$ & -1.40 \\
\hline & & $\log \mathrm{CFU} / \mathrm{g}$ & 7.49 & \pm & 0.13 & $\mathrm{bc}$ & nd & & & & 8.87 & \pm & 0.04 & $a b$ & 1.38 \\
\hline \multirow[t]{4}{*}{ Lp. plantarum } & A310 & & & & & & & & & & & & & & \\
\hline & & $\mathrm{pH}$ & 6.86 & \pm & 0.00 & $\mathrm{a}$ & 6.37 & \pm & 0.01 & $a b$ & 4.42 & \pm & 0.11 & $a b c$ & 2.44 \\
\hline & & TTA & 0.15 & \pm & 0.00 & $\mathrm{e}$ & 0.28 & \pm & 0.04 & & 1.63 & \pm & 0.25 & $a b c$ & -1.48 \\
\hline & & $\log \mathrm{CFU} / \mathrm{g}$ & 7.31 & \pm & 0.02 & $\mathrm{bc}$ & nd & & & & 8.26 & \pm & 0.21 & $\mathrm{bc}$ & 0.95 \\
\hline \multirow[t]{4}{*}{ Lp. plantarum } & B11 & & & & & & & & & & & & & & \\
\hline & & $\mathrm{pH}$ & 6.80 & \pm & 0.01 & $a b c$ & 6.55 & \pm & 0.02 & $a b$ & 4.60 & \pm & 0.11 & $a b c$ & 2.20 \\
\hline & & TTA & 0.18 & \pm & 0.04 & de & 0.23 & \pm & 0.04 & & 1.20 & \pm & 0.07 & $a b c$ & -1.20 \\
\hline & & $\log \mathrm{CFU} / \mathrm{g}$ & 7.33 & \pm & 0.04 & $\mathrm{bc}$ & nd & & & & 8.30 & \pm & 0.06 & $\mathrm{c}$ & 0.97 \\
\hline \multirow[t]{4}{*}{ Lp. plantarum } & B15 & & & & & & & & & & & & & & \\
\hline & & $\mathrm{pH}$ & 6.72 & \pm & 0.09 & $a b c$ & 5.74 & \pm & 0.09 & $a b c$ & 4.21 & \pm & 0.01 & $a b c$ & 2.51 \\
\hline & & TTA & 0.18 & \pm & 0.04 & de & 0.50 & \pm & 0.07 & & 1.50 & \pm & 0.00 & $a b c$ & -1.32 \\
\hline & & $\log \mathrm{CFU} / \mathrm{g}$ & 7.13 & \pm & 0.07 & $\mathrm{c}$ & nd & & & & 8.47 & \pm & 0.01 & $a b$ & 1.34 \\
\hline \multirow[t]{4}{*}{ Lp. plantarum } & B18 & & & & & & & & & & & & & & \\
\hline & & $\mathrm{pH}$ & 6.72 & \pm & 0.04 & $a b c$ & 5.82 & \pm & 0.11 & $a b c$ & 4.24 & \pm & 0.08 & $a b c$ & 2.48 \\
\hline & & TTA & 0.15 & \pm & 0.00 & $\mathrm{e}$ & 0.45 & \pm & 0.07 & & 1.53 & \pm & 0.11 & $a b c$ & -1.38 \\
\hline & & $\log \mathrm{CFU} / \mathrm{g}$ & 7.36 & \pm & 0.03 & $\mathrm{bc}$ & nd & & & & 8.37 & \pm & 0.13 & $a b$ & 1.01 \\
\hline \multirow[t]{4}{*}{ Lp. plantarum } & B19 & & & & & & & & & & & & & & \\
\hline & & $\mathrm{pH}$ & 6.64 & \pm & 0.02 & $a b c$ & 6.17 & \pm & 0.47 & $a b$ & 4.51 & \pm & 0.41 & $a b c$ & 2.13 \\
\hline & & TTA & 0.25 & \pm & 0.00 & abcde & 0.38 & \pm & 0.18 & & 1.40 & \pm & 0.35 & $a b c$ & -1.15 \\
\hline & & $\log \mathrm{CFU} / \mathrm{g}$ & 7.37 & \pm & 0.13 & $\mathrm{bc}$ & nd & & & & 8.31 & \pm & 0.28 & $\mathrm{bc}$ & 0.94 \\
\hline \multirow[t]{4}{*}{ Lp. plantarum } & B21 & & & & & & & & & & & & & & \\
\hline & & $\mathrm{pH}$ & 6.72 & \pm & 0.04 & $a b c$ & 6.00 & \pm & 0.11 & $a b$ & 4.24 & \pm & 0.03 & $a b c$ & 2.48 \\
\hline & & TTA & 0.23 & \pm & 0.04 & bcde & 0.43 & \pm & 0.04 & & 1.65 & \pm & 0.00 & $a b c$ & -1.42 \\
\hline & & $\log \mathrm{CFU} / \mathrm{g}$ & 7.41 & \pm & 0.02 & $\mathrm{bc}$ & nd & & & & 8.38 & \pm & 0.00 & $\mathrm{c}$ & 0.97 \\
\hline \multirow[t]{4}{*}{ Lp. plantarum } & B23 & & & & & & & & & & & & & & \\
\hline & & $\mathrm{pH}$ & 6.63 & \pm & 0.06 & $a b c$ & 5.67 & \pm & 0.01 & $a b c$ & 4.35 & \pm & 0.02 & $a b c$ & 2.28 \\
\hline & & TTA & 0.20 & \pm & 0.00 & cde & 0.55 & \pm & 0.00 & & 1.48 & \pm & 0.04 & $a b c$ & -1.28 \\
\hline & & $\log \mathrm{CFU} / \mathrm{g}$ & 7.34 & \pm & 0.48 & $\mathrm{bc}$ & nd & & & & 8.52 & \pm & 0.01 & $a b$ & 1.18 \\
\hline \multirow[t]{4}{*}{ Lp.plantarum } & B27 & & & & & & & & & & & & & & \\
\hline & & $\mathrm{pH}$ & 6.76 & \pm & 0.03 & $a b c$ & 6.59 & \pm & 0.01 & $\mathrm{a}$ & 4.68 & \pm & 0.05 & $a b c$ & 2.08 \\
\hline & & TTA & 0.30 & \pm & 0.00 & abcd & 0.33 & \pm & 0.04 & & 1.08 & \pm & 0.04 & c & -0.78 \\
\hline & & $\log \mathrm{CFU} / \mathrm{g}$ & 9.20 & \pm & 0.00 & $\mathrm{a}$ & nd & & & & 11.06 & \pm & 0.01 & $\mathrm{a}$ & 1.86 \\
\hline \multirow[t]{4}{*}{ Lc. casei } & B28 & & & & & & & & & & & & & & \\
\hline & & $\mathrm{pH}$ & 6.63 & \pm & 0.05 & $a b c$ & 5.97 & \pm & 0.04 & $\mathrm{abc}$ & 4.64 & \pm & 0.13 & $a b c$ & 1.99 \\
\hline & & TTA & 0.33 & \pm & 0.04 & $a b c$ & 0.53 & \pm & 0.04 & & 1.25 & \pm & 0.14 & $\mathrm{bc}$ & -0.92 \\
\hline & & $\log \mathrm{CFU} / \mathrm{g}$ & 7.84 & \pm & 0.07 & $a b$ & nd & & & & 9.84 & \pm & 0.01 & $a b$ & 2.00 \\
\hline Lp. plantarum & B29 & & & & & & & & & & & & & & \\
\hline & & $\mathrm{pH}$ & 6.56 & \pm & 0.04 & $\mathrm{c}$ & 5.69 & \pm & 0.07 & $a b c$ & 4.15 & \pm & 0.07 & $\mathrm{c}$ & 2.41 \\
\hline
\end{tabular}


Table 2 (continued)

\begin{tabular}{|c|c|c|c|c|c|c|c|c|c|c|c|c|c|c|c|}
\hline \multicolumn{2}{|l|}{$\mathrm{LAB}$ isolate } & \multicolumn{5}{|c|}{$0 \mathrm{~h}$} & \multicolumn{4}{|l|}{$4 \mathrm{~h}$} & \multicolumn{4}{|l|}{$7 \mathrm{~h}$} & \multirow{2}{*}{$\frac{\text { Dynamics }}{-0.90}$} \\
\hline & & TTA & 0.35 & \pm & 0.00 & $a b$ & 0.50 & \pm & 0.00 & & 2.18 & \pm & 0.39 & $\mathrm{a}$ & \\
\hline & & $\log \mathrm{CFU} / \mathrm{g}$ & 7.72 & \pm & 0.12 & $a b c$ & nd & & & & 9.59 & \pm & 0.23 & $a b$ & 1.87 \\
\hline \multirow[t]{4}{*}{ Lp. plantarum } & B32 & & & & & & & & & & & & & & \\
\hline & & $\mathrm{pH}$ & 6.60 & \pm & 0.05 & $\mathrm{bc}$ & 5.70 & \pm & 0.07 & $a b c$ & 4.19 & \pm & 0.01 & $\mathrm{c}$ & 2.41 \\
\hline & & TTA & 0.35 & \pm & 0.07 & $a b$ & 0.63 & \pm & 0.18 & & 1.83 & \pm & 0.11 & $a b$ & -1.48 \\
\hline & & $\log \mathrm{CFU} / \mathrm{g}$ & 7.63 & \pm & 0.04 & $a b c$ & nd & & & & 8.37 & \pm & 0.16 & $a b$ & 0.74 \\
\hline \multirow{4}{*}{ Lc. casei } & B33 & & & & & & & & & & & & & & \\
\hline & & $\mathrm{pH}$ & 6.61 & \pm & 0.01 & $a b c$ & 5.98 & \pm & 0.09 & $a b$ & 4.82 & \pm & 0.08 & $a b c$ & 1.79 \\
\hline & & TTA & 0.25 & \pm & 0.00 & abcde & 0.53 & \pm & 0.18 & & 1.23 & \pm & 0.46 & $a b c$ & -0.98 \\
\hline & & $\log \mathrm{CFU} / \mathrm{g}$ & 7.69 & \pm & 0.01 & $a b c$ & nd & & & & 9.44 & \pm & 0.13 & $\mathrm{ab}$ & 1.75 \\
\hline \multirow[t]{4}{*}{ Lp. plantarum } & C11 & & & & & & & & & & & & & & \\
\hline & & $\mathrm{pH}$ & 6.55 & \pm & 0.00 & $\mathrm{c}$ & 5.64 & \pm & 0.08 & $\mathrm{abc}$ & 4.14 & \pm & 0.01 & $\mathrm{c}$ & 2.41 \\
\hline & & TTA & 0.35 & \pm & 0.00 & $a b$ & 0.60 & \pm & 0.00 & & 2.20 & \pm & 0.35 & $\mathrm{a}$ & -1.85 \\
\hline & & $\log \mathrm{CFU} / \mathrm{g}$ & 7.74 & \pm & 0.10 & $a b c$ & nd & & & & 9.47 & \pm & 0.13 & $\mathrm{ab}$ & 1.73 \\
\hline \multirow[t]{5}{*}{ Lp. plantarum } & $\mathrm{C12}$ & & & & & & & & & & & & & & \\
\hline & & $\mathrm{pH}$ & 6.65 & \pm & 0.01 & $a b c$ & 6.05 & \pm & 0.02 & $a b$ & 4.26 & \pm & 0.07 & $a b c$ & 2.39 \\
\hline & & TTA & 0.40 & \pm & 0.00 & a & 0.48 & \pm & 0.04 & & 2.38 & \pm & 0.04 & $\mathrm{a}$ & -1.98 \\
\hline & & $\log \mathrm{CFU} / \mathrm{g}$ & 7.93 & \pm & 0.02 & $a b c$ & nd & & & & 9.40 & \pm & 1133.00 & $\mathrm{ab}$ & 1.47 \\
\hline & & Growth $\log C F U / g$ & & & & & & & & & 1.47 & & & & \\
\hline \multirow[t]{5}{*}{ Lp. plantarum } & C17 & & & & & & & & & & & & & & \\
\hline & & $\mathrm{pH}$ & 6.63 & \pm & 0.03 & $a b c$ & 6.37 & \pm & 0.01 & $a b$ & 4.72 & \pm & 0.23 & $a b c$ & 1.91 \\
\hline & & TTA & 0.40 & \pm & 0.00 & $\mathrm{a}$ & 0.45 & \pm & 0.00 & & 1.38 & \pm & 0.11 & $a b c$ & -0.98 \\
\hline & & $\log \mathrm{CFU} / \mathrm{g}$ & 7.75 & \pm & 0.07 & $a b c$ & nd & & & & 9.89 & \pm & 0.21 & $\mathrm{ab}$ & 2.14 \\
\hline & & Growth log CFU/g & & & & & & & & & 2.14 & & & & \\
\hline \multirow[t]{5}{*}{ Lp. plantarum } & $\mathrm{C} 28$ & & & & & & & & & & & & & & \\
\hline & & $\mathrm{pH}$ & 6.81 & \pm & 0.02 & $a b c$ & 6.07 & \pm & 0.01 & $a b$ & 4.53 & \pm & 0.18 & $a b c$ & 2.28 \\
\hline & & TTA & 0.20 & \pm & 0.00 & cde & 0.30 & \pm & 0.00 & & 1.48 & \pm & 0.25 & $a b c$ & -1.28 \\
\hline & & $\log \mathrm{CFU} / \mathrm{g}$ & 7.58 & \pm & 0.54 & $\mathrm{bc}$ & nd & & & & 9.35 & \pm & 0.01 & $a b$ & 1.77 \\
\hline & & Growth log CFU/g & & & & & & & & & 1.77 & & & & \\
\hline \multirow[t]{7}{*}{ Lp. plantarum } & C38 & & & & & & & & & & & & & & \\
\hline & & $\mathrm{pH}$ & 6.75 & \pm & 0.01 & $a b c$ & 5.93 & \pm & 0.04 & $a b$ & 4.41 & \pm & 0.04 & $a b c$ & 2.34 \\
\hline & & TTA & 0.25 & \pm & 0.00 & abcde & 0.40 & \pm & 0.00 & & 1.18 & \pm & 0.04 & $a b c$ & -0.93 \\
\hline & & $\log \mathrm{CFU} / \mathrm{g}$ & 7.70 & \pm & 0.33 & $a b c$ & nd & & & & 9.58 & \pm & 0.02 & $a b$ & 1.88 \\
\hline & & Average $p H$ decrease & & & & & & & & & & & & & 2.25 \\
\hline & & Average TTA increas & & & & & & & & & & & & & -1.22 \\
\hline & & Average growth log $C$ & $C F U / g$ & & & & & & & & & & & & 1.47 \\
\hline
\end{tabular}

Values are expressed as the mean \pm SD from triplicate determinations. Abbreviations: $n d$ Not determined. Different letters indicate statistical difference related to the different characteristics of LAB isolates (viable cell-counts, $\mathrm{pH}$ and total titratable acidity) at specific time ( $0 \mathrm{~h}, 4 \mathrm{~h}$, or $7 \mathrm{~h}$ ) using least significant difference test $(P<0.05)$. $P$-values were adjusted using Bonferroni's method

B15 and C28, Table 3). In contrast, when using casein agar, seven $\mathrm{LAB}$ isolates were able to degrade casein in plate assay (Lp. plantarum A16, A25, B18, B23, C11, C38 and Lc. casei A23, Table 3).

\section{Selected LAB isolates displayed single, double and multiple antibiotic resistance}

Ampicillin resistance was identified for all LAB isolates regardless of the time of incubation, except for one strain ( $L c$. casei A23, Table 4). Interestingly, five LAB isolates displayed a double antibiotic susceptibility after $24 \mathrm{~h}$ (Table 4) while, after $48 \mathrm{~h}$, all LAB isolates were resistant to Amp and Cli. Multiple antibiotic resistance was detected in $3 \mathrm{LAB}$ isolates after $48 \mathrm{~h}$ (Table 4 ). 


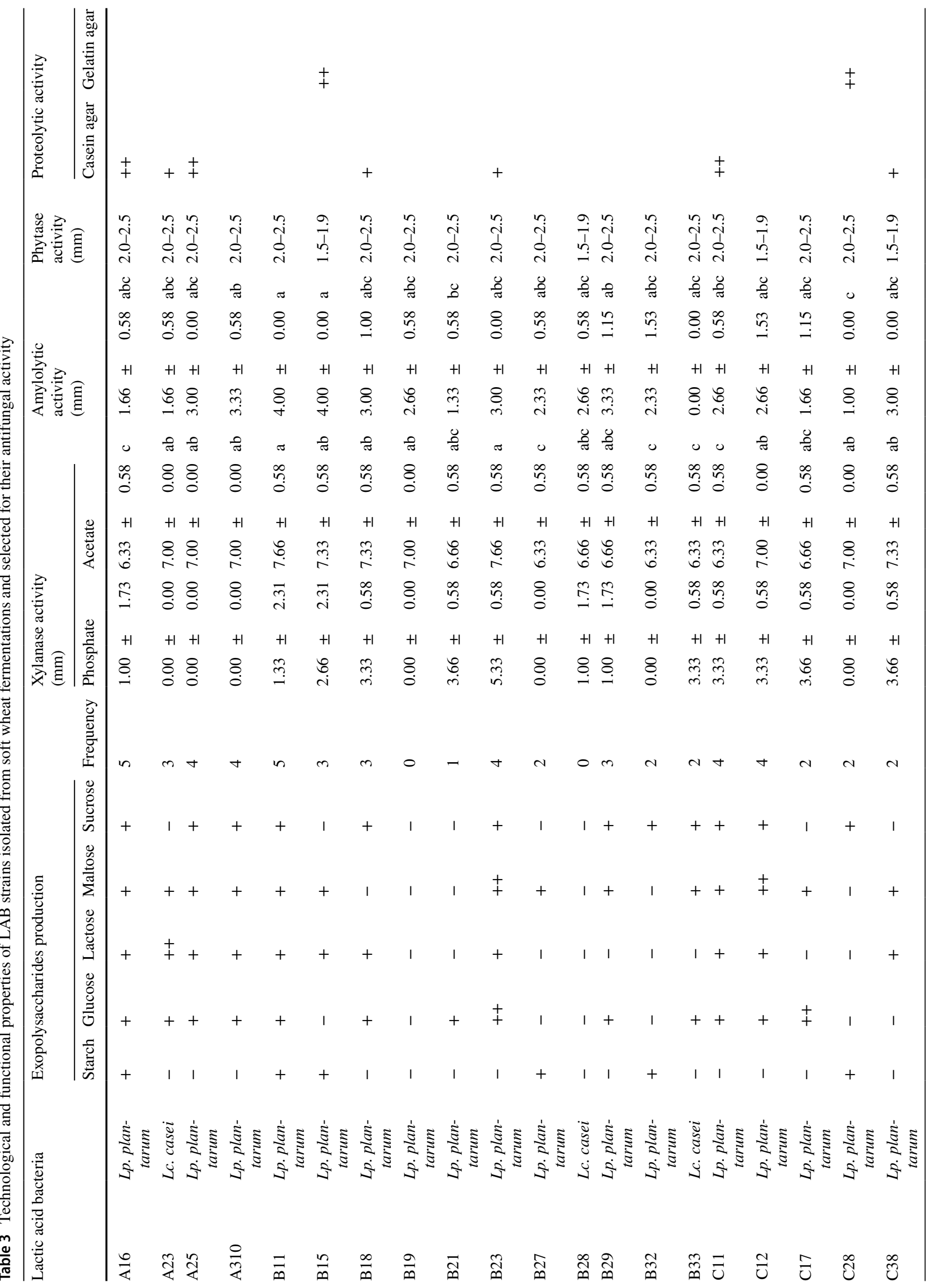


Table 4 Antibiotic resistance of LAB strains selected for their antifungal, technological and functional activities

\begin{tabular}{llll}
\hline \multirow{2}{*}{ Lactic acid bacteria } & \multicolumn{2}{l}{ Antibiotic resistance } \\
\cline { 3 - 4 } & & $24 \mathrm{~h}$ & $48 \mathrm{~h}$ \\
\hline A16 & Lp. plantarum & Amp/Cli & Amp/Cli \\
A23 & Lc. casei & Ery/Tet & Ery/Cli/Tet \\
A25 & Lp. plantarum & Amp & Amp/Cli \\
A310 & Lp. plantarum & Amp/Cli & Amp/Gen/Cli \\
B11 & Lp. plantarum & Amp/Cli & Amp/Cli \\
B15 & Lp. plantarum & Amp & Amp/Cli \\
B18 & Lp. plantarum & Amp/Cli & Amp/Cli/Chl \\
\hline
\end{tabular}

LAB with MIC higher than EFSA breakpoints are considered as resistant strains

\section{Discussions}

A. niger is a spoilage fungus known to grow rapidly on the surface of bakery products (Smith, Daifas, El-Khoury, Koukoutsis, \& El-Khoury, 2004). Previous studies have shown that different LAB strains (Lp. plantarum CE42, CE60, CE84, Ll. curvatus CE83 and Pediococcus pentosaceus CE65 and CE23), isolated from wheat bran sourdough, have a strong antifungal activity towards A. niger and Aspergillus oryzae (Manini et al. 2016). In this regard, our study showed the ability of $L p$. plantarum isolates to inhibit in vitro the growth not only of Aspergillus species but also of a broad range of filamentous fungi (16 molds tested) which were chosen for their spoilage potential in bread and bakery goods, and/or for their potential to produce mycotoxins.

The antifungal potential differed between LAB isolates, and our results showed that the inhibition of different fungal species was strain dependent. Similar inhibition activity was observed elsewhere in Ll. brevis but not in Lp. plantarum (Manini et al. 2016; Ruiz Rodríguez et al. 2016). However, the antifungal effect strain dependency of $L p$. plantarum has been observed from other authors in isolates from different food matrices (Russo et al. 2017). In terms of biocontrol potential, the application of different LAB strains in food processing could expand horizons for preventing food spoilage. The capability of $L p$. plantarum to prevent spoilage has been linked to the direct competition for growth substrates between this bacteria and spoilage fungi, and to the production of compounds such as lactic acid, acetic acid, hydrogen peroxide, phenyllactic acid and bacteriocins (Cortés-Zavaleta et al. 2014; Gupta and Srivastava 2014). In the present research, the nature of LAB antifungal strenght (organic acid and/or proteinaceous metabolites) was not investigated. Some authors highlighted the major contribute of organic acids for antifungal activity in LAB strains and identified phenyllactic acid as one of the most effective antifungal compounds in Lp. plantarum, showing that 
its production is strain-dependent (Dal Bello et al. 2007; Schnurer and Magnussoon 2007). The disadvantages of studying antifungal activity based on colony growth in synthetic selective media are well known and include that the concentration of glucose seems to affect antifungal activity and MRS medium could stimulate the production of antifungal compounds (Fraberger et al. 2020). Further research is needed to assess the impact of using LAB strains as a starter cultures in sourdough as antifungal controlling agents.

The ability of LAB to decrease the $\mathrm{pH}$ during sourdough fermentation is an important technological feature of this bacterial group. Recently, differences in the growth and acidification rate between different LAB strains have been reported (Manini et al. 2016; Ruiz Rodríguez et al. 2016). In this study, we also observed a significant difference in $\mathrm{LAB}$ growth and acidification rate between isolates, further confirming previous study observation. Noteworthy, LAB isolates showed lower population growth (final LAB growth range between 8.30 and $9.89 \log \mathrm{CFU} / \mathrm{g}$ range) compared with previous researches $(11 \log \mathrm{CFU} / \mathrm{g})$ (Manini et al., 2016). The capability of LAB isolates to reduce the $\mathrm{pH}$ to about 4 , after 7 or $8 \mathrm{~h}$, is an important characteristic to obtain bread and bakery products with better rheological and flavor characteristics. In our study, the highest $\mathrm{pH}$ values were showed particularly by $L c$. casei strains, as observed by other authors (Paucean et al. 2013).

Concerning the technological properties, such as EPS and enzymatic activities, of the LAB isolates. EPS such as glucans and/or fructans play an important role in the texture, taste perception and stability of fermented food (Tieking and Gänzle 2005; Galle and Arendt 2014). Previous studies suggested that at least one or more EPS producing bacterial strain is found in sourdough microbiota (Tieking, et al. 2003; Manini et al. 2016). According to Manini et al. (2016), one of the major EPS producers was Lp. plantarum. The differences in EPS production found in our study between LAB isolates indicates that EPS production is largely straindependent. On the other hand, xylanase and phytase are used to increase dough viscosity, bread volume, shelf life and improve mineral bioavailability in bakery products (Poutanen et al. 2009). The ability of all LAB isolates tested in our study to degrade hexacalcium phytate is consistent with previous studies (De Angelis et al. 2003; Manini et al. 2016).

The degradation of peptides and proteins by LAB contributes to flavor, antimicrobial activity, and structure of different foods. In our study, the proteolysis evaluation resulted in scarce protein degradation, in agreement with previous findings. Only a LAB strain belonging to Lactococcus lactis species and isolated from quinoa sourdough fermentation showed strong proteinase activity (Ruiz Rodríguez et al. 2016). Furthermore, our results indicate different proteolytic capacities between LAB isolates, in accordance elsewhere (Alfonzo et al. 2013). Differences in the ability to utilize gelatin or casein may be related to the differences in the substrate specificities of the enzyme produced. A comparative genome analysis of several Lp. plantarum strains demonstrated the versatility to acquire and retained functional capacities (Siezen et al. 2010) but lack of environmental adaptation (Martino et al. 2016). Recently, the capacity of Lp. plantarum isolated from cassava to synthesize EPS, metabolize carbohydrate (starch), polyphenols and vitamin $\mathrm{B}$ has been demonstrated through genomics and transcriptomics (Turpin et al. 2018). Further research is needed to find the link between gene content and functional properties of different LAB strains in food and their interactions.

The microbial resistance to antibiotics represents a concern for public health. $L p$. plantarum and $L c$. casei here tested are included in the Qualified Presumption of Safety (QPS) list of the European Food Safety Authority (EFSA 2007). However, all Lp. plantarum isolates assayed for antibiotics sensitivity, showing susceptibility to ampicillin and clindamycin after $48 \mathrm{~h}$, contrarily to what described for Lp. plantarum strains isolated from quinoa sourdoughs and wheat bran elsewhere (Manini et al. 2016; Ruiz Rodríguez et al. 2016). Noteworthy, among Lp. plantarum we observed that two isolates showed an antibiotic multi-resistance. Differences in the antibiotic resistance between LAB isolates may be attributed to the non-specific mechanism of multidrug transporters, general stress-induced response, a mutation on penicillin-binding proteins (Delgado et al. 2005), or defective autolytic enzyme activity (Kim et al. 1982). In our study, two LAB isolates were susceptible to chloramphenicol, erythromycin, and tetracycline, confirming that lactobacilli are usually resistant to antibiotics that inhibit the synthesis of proteins (Danielsen and Wind 2003; Shao et al. 2015). Further investigation is needed to explore if this antibiotic resistance is non-transmissible.

\section{Conclusions}

In conclusion, the antifungal activity of the different $\mathrm{LAB}$ strains isolated from type "0" soft wheat flour sourdough inhibited the growth of several fungal species in vitro. The LAB strains used in this study demonstrated a different antifungal potential, EPS production, and enzymatic activity. $L p$. plantarum A16, A25, B11, and B15 reduced the growth of several fungal species and showed the greatest capabilities to growth in different carbon sources. These results demonstrated that type "0" soft wheat flour sourdough is a good source to isolate LAB strains with potential for future food applications, as natural bio-control agents to inhibit fungal growth and most probably to extend the shelf life of bakery products. Care should be taken with the selection of LAB strains resistant to ampicillin and clindamycin. Further study is recommended to optimize the conditions to increase the 
antifungal activity for food application in greening the food processing industry.

Acknowledgement The authors thank Maina Company for providing sourdough samples from which some of the lactic strains studied were isolated.

Author contributions PD: Conceived and designed study, Wrote the paper; $J M-G$ : Performed research, Analyzed data, Wrote the paper; $I F$ : Performed research; MR: Performed research. All authors have read and agree to be a published version of the final version of the manuscript.

Funding Open access funding provided by Università degli Studi di Torino within the CRUI-CARE Agreement. This research did not receive any specific grant from funding agencies in the public, commercial, or not-for-profit sectors.

Data availability The authors declare data transparency.

Code availability Not applicable.

\section{Declarations}

Conflict of interest The authors declare no conflict of interest.

Ethical approval This article does not contain any studies with human participants or animals experiments.

Open Access This article is licensed under a Creative Commons Attribution 4.0 International License, which permits use, sharing, adaptation, distribution and reproduction in any medium or format, as long as you give appropriate credit to the original author(s) and the source, provide a link to the Creative Commons licence, and indicate if changes were made. The images or other third party material in this article are included in the article's Creative Commons licence, unless indicated otherwise in a credit line to the material. If material is not included in the article's Creative Commons licence and your intended use is not permitted by statutory regulation or exceeds the permitted use, you will need to obtain permission directly from the copyright holder. To view a copy of this licence, visit http://creativecommons.org/licenses/by/4.0/.

\section{References}

Alfonzo A, Ventimiglia G, Corona O, Di Gerlando R, Gaglio R, Francesca $\mathrm{N}$ et al (2013) Diversity and technological potential of lactic acid bacteria of wheat flours. Food Microbiol 36(2):343-354. https://doi.org/10.1016/j.fm.2013.07.003

Anastasio M, Pepe O, Cirillo T, Palomba S, Blaiotta G, Villani F (2010) Selection and use of phytate-degrading LAB to improve cerealbased products by mineral solubilization during dough fermentation. J Food Sci 75:28-35. doi 10.1111/j.1750 3841.2009.01402.x

Arena MP, Russo P, Spano G, Capozzi V (2019) Exploration of the microbial biodiversity associated with North Apulian sourdoughs and the effect of the increasing number of inoculated lactic acid bacteria strains on the biocontrol against fungal spoilage. Fermentation. https://doi.org/10.3390/fermentation5040097

Bennet RN, Wallsgrove RM (1994) Secondary metabolites in plant defence mechanisms. New Phytol 127(4):617-633. https://doi.org/ 10.1111/j.1469-8137.1994.tb02968.x
Berthier F, Ehrlich SD (1998) Rapid species identification within two groups of closely related lactobacilli using PCR primers that target the 16S/23S rRNA spacer region. FEMS Microbiol Lett 161:97-106. https://doi.org/10.1111/j.1574-6968.1998.tb12934.x

Cortés-Zavaleta O, López-Malo A, Hernández-Mendoza A, García HS (2014) Antifungal activity of lactobacilli and its relationship with 3-phenyllactic acid production. Int J Food Microbiol 173:30-35. https://doi.org/10.1016/j.ijfoodmicro.2013.12.016

Crawley MJ (2007) The R book. Wiley, West Sussex

Dal Bello F, Clarke CI, Ryan LAM, Ulmer H, Schober TJ, Strom K, Arendt EK (2007) Improvement of the quality and shelf life of wheat bread by fermentation with the antifungal strain Lactobacillus plantarum FST 1.7. J Cereal Sci 45:309-318. https://doi.org/ 10.1016/j.jcs.2006.09.004

Danielsen M, Wind A (2003) Susceptibility of Lactobacillus spp. to antimicrobial agents. Int J Food Microbiol 82(1):1-11. https://doi. org/10.1016/S0168-1605(02)00254-4

De Angelis M, Gallo G, Corbo MR, McSweeney PLH, Faccia M, Giovine M, Gobbetti M (2003) Phytase activity in sourdough lactic acid bacteria: purification and characterization of a phytase from Lactobacillus sanfranciscensis CB1. Int J Food Microbiol 87(3):259-270. https://doi.org/10.1016/S01681605(03)00072-2

De Vuyst L, Weckx S (2010) The functional role of lactic acid bacteria in cocoa bean fermentation. In: Mozzi F, Raya RR, Vignolo GM (eds) Biotechnology of lactic acid bacteria: novel applications, 2nd edn. Wiley, New York, pp 301-325

Delgado S, Flórez AB, Mayo B (2005) Antibiotic susceptibility of Lactobacillus and Bifidobacterium species from the human gastrointestinal tract. Curr Microbiol 50(4):202-207. https://doi.org/ 10.1007/s00284-004-4431-3

EFSA (2007) Introduction of a qualified presumption of safety (QPS) approach for assessment of selected microorganisms referred to EFSA. EFSA J 587:1-16. https://doi.org/10.2903/j.efsa.2007.587

EFSA (2012) Guidance on the assessment of bacterial susceptibility to antimicrobials of human and veterinary importance. EFSA J 10(6):1-10. https://doi.org/10.2903/j.efsa.2012.2740

Fekri A, Torbati M, Yari Khosrowshahi A, Bagherpour Shamloo H, Azadmard-Damirchi S (2020) Functional effects of phytatedegrading, probiotic lactic acid bacteria and yeast strains isolated from Iranian traditional sourdough on the technological and nutritional properties of whole wheat bread. Food Chem. https://doi. org/10.1016/j.foodchem.2019.125620

Fraberger V, Ammer C, Domig KJ (2020) Functional properties and sustainability improvement of sourdough bread by lactic acid bacteria. Microorganisms 8(12):1-17. https://doi.org/10.3390/micro organisms 8121895

Galle S, Arendt EK (2014) Exopolysaccharides from sourdough lactic acid bacteria. Crit Rev Food Sci Nutr 54(7):891-901. https://doi. org/10.1080/10408398.2011.617474

Gänzle MG, Zheng J (2019) Lifestyles of sourdough lactobacilli - do they matter for microbial ecology and bread quality? Int J Food Microbiol 302:15-23. https://doi.org/10.1016/j.ijfoodmicro.2018. 08.019

Garcia MV, Bernardi AO, Copetti MV (2019) The fungal problem in bread production: insights of causes, consequences, and control methods. Curr Opin Food Sci 29:1-6. https://doi.org/10.1016/j. cofs.2019.06.010

Garofalo C, Zannini E, Aquilanti L, Silvestri G, Fierro O, Picariello G, Clementi F (2012) Selection of sourdough lactobacilli with antifungal activity for use as biopreservatives in bakery products. J Agric Food Chem 60(31):7719-7728. https://doi.org/10.1021/ jf301173u

Gupta R, Srivastava S (2014) Antifungal effect of antimicrobial peptides (AMPs LR14) derived from Lactobacillus plantarum strain 
$\mathrm{LR} / 14$ and their applications in prevention of grain spoilage. Food Microbiol 42:1-7. https://doi.org/10.1016/j.fm.2014.02.005

Kim KS, Morrison JO, Bayer AS (1982) Deficient autolytic enzyme activity in antibiotic-tolerant lactobacilli. Infect Immun 36(2):582-585. https://doi.org/10.1128/iai.36.2.582-585.1982

Legan JD (1993) Mould spoilage of bread: the problem and some solutions. Int Biodeterior Biodegradation 32(1-3):33-53. https://doi. org/10.1016/0964-8305(93)90038-4

Magnusson J, Schnürer J (2001) Lactobacillus coryniformis subsp. coryniformis strain $\mathrm{Si} 3$ produces a broad-spectrum proteinaceous antifungal compound. Appl Environ Microbiol 67(1):1-5. https:// doi.org/10.1128/AEM.67.1.1

Manini F, Casiraghi MC, PoutanennK, Brasca M, Erba D, Plumed-Ferrer C (2016) Characterization of lactic acid bacteria isolated from wheat bran sourdough. LWT - Food Sci Technol 66:275-283. https://doi.org/10.1016/j.lwt.2015.10.045

Martino ME, Bayjanov JR, Caffrey BE et al (2016) Nomadic lifestyle of Lactobacillus plantarum revealed by comparative genomics of 54 strains isolated from different habitats. Environ Microbiol 18(12):4974-4989. https://doi.org/10.1111/1462-2920.13455

Minervini F, De Angelis M, Di Cagno R, Pinto D, Siragusa S, Rizzello CG, Gobbetti M (2010) Robustness of Lactobacillus plantarum starters during daily propagation of wheat flour sourdough type I. Food Microbiol 27(7):897-908. https://doi.org/10.1016/j.fm. 2010.05.021

Nionelli L, Curri N, Curiel JA et al (2014) Exploitation of Albanian wheat cultivars: characterization of the flours and lactic acid bacteria microbiota, and selection of starters for sourdough fermentation. Food Microbiol 44:96-107. https://doi.org/10.1016/j.fm. 2014.05.011

Paucean A, Vodnar DC, Socaci SA, Socaciu C (2013) Carbohydrate metabolic conversions to lactic acid and volatile derivatives, as influenced by Lactobacillus plantarum ATCC 8014 and Lactobacillus casei ATCC 393 efficiency during in vitro and sourdough fermentation. Eur Food Res Technol 237(5):679-689. https://doi. org/10.1007/s00217-013-2042-6

Poutanen K, Flander L, Katina K (2009) Sourdough and cereal fermentation in a nutritional perspective. Food Microbiol 26(7):693-699. https://doi.org/10.1016/j.fm.2009.07.011

Reale A, Di Renzo T, Succi M, Tremonte P, Coppola R, Sorrentino E (2013) Microbiological and fermentative properties of baker's yeast starter used in breadmaking. J Food Sci. https://doi.org/10. 1111/1750-3841.12206

Rosenkvist H, Hansen $\AA$ (1995) Contamination profiles and characterisation of Bacillus species in wheat bread and raw materials for bread production. Int J Food Microbiol 26(3):353-363. https:// doi.org/10.1016/0168-1605(94)00147-X

Ruiz Rodríguez L, Vera Pingitore E, Rollan G et al (2016) Biodiversity and technological-functional potential of lactic acid bacteria isolated from spontaneously fermented quinoa sourdoughs. J Appl Microbiol 120(5):1289-1301. https://doi.org/10.1111/jam.13104

Russo P, Arena MP, Fiocco D, Capozzi V, Drider D, Spano G (2017) Lactobacillus plantarum with broad antifungal activity: A promising approach to increase safety and shelf-life of cereal-based products. Int J Food Microbiol 247:48-54. https://doi.org/10.1016/j. ijfoodmicro.2016.04.027

Sadeghi A, Ebrahimi M, Mortazavi SA, Abedfar A (2019a) Application of the selected antifungal LAB isolate as a protective starter culture in pan whole-wheat sourdough bread. Food Control. https:// doi.org/10.1016/j.foodcont.2018.08.013

Sadeghi A, Ebrahimi M, Raeisi M, Nematollahi Z (2019b) Biological control of foodborne pathogens and aflatoxins by selected probiotic LAB isolated from rice bran sourdough. Biol Control 130:70-79. https://doi.org/10.1016/j.biocontrol.2018.09.017
Salmenkallio-Marttila M, Katina K, Autio K (2001) Effects of bran fermentation on quality and microstructure of high-fiber wheat bread. Cereal Chem 78(4):429-435. https://doi.org/10.1094/ CCHEM.2001.78.4.429

Schnurer J, Magnussoon J (2007) Antifungal lactic acid bacteria as biopreservatives. Trends Food Sci Technol 16:70-78. https://doi. org/10.1016/j.tifs.2004.02.014

Shao Y, Zhang W, Guo H, Pan L, Zhang H, Sun T (2015) Comparative studies on antibiotic resistance in Lactobacillus casei and Lactobacillus plantarum. Food Control 50:250-258. https://doi. org/10.1016/j.foodcont.2014.09.003

Siezen RJ, Tzeneva VA, Castioni A et al (2010) Phenotypic and genomic diversity of Lactobacillus plantarum strains isolated from various environmental niches. Environ Microbiol 12(3):758 773. https://doi.org/10.1111/j.1462-2920.2009.02119.x

Smith JP, Daifas DP, El-Khoury W, Koukoutsis J, El-Khoury A (2004) Shelf life and safety concerns of bakery products - A Review. Crit Rev Food Sci Nutr 44(1):19-55. https://doi.org/10.1080/10408 690490263774

Taccari M, Aquilanti L, Polverigiani S, Osimani A, Garofalo C, Milanović V, Clementi F (2016) Microbial diversity of type I sourdoughs prepared and back-slopped with wholemeal and refined soft (Triticum aestivum) wheat flours. J Food Sci 81(8):19962005. https://doi.org/10.1111/1750-3841.13372

Teymoortash A, Wollstein AC, Lippert BM, Peldszus R, Werner JA (2002) Bacteria and pathogenesis of human salivary calculus. Acta Oto-Laryngol 122:210-214. https://doi.org/10.1080/00016 480252814252

Tieking M, Gänzle MG (2005) Exopolysaccharides from cereal-associated lactobacilli. Trends Food Sci Tech 16(1-3):79-84. https:// doi.org/10.1016/j.tifs.2004.02.015

Tieking M, Korakli M, Ehrmann MA, Gänzle MG, Vogel RF (2003) In situ production of exopolysaccharides during sourdough fermentation by cereal and intestinal isolates of lactic acid bacteria. Appl Environ Microbiol 69(2):945-952. https://doi.org/10.1128/ AEM.69.2.945-952.2003

Torrieri E, Pepe O, Ventorino V, Masi P, Cavella S (2014) Effect of sourdough at different concentrations on quality and shelf life of bread. LWT - Food Sci Technol 56(2):508-516. https://doi.org/ 10.1016/j.1wt.2013.12.005

Turpin W, Weiman M, Guyot JP, Lajus A, Cruveiller S, Humblot C (2018) The genomic and transcriptomic basis of the potential of Lactobacillus plantarum A6 to improve the nutritional quality of a cereal based fermented food. Int J Food Microbiol 266:346-354. https://doi.org/10.1016/j.ijfoodmicro.2017.10.011

Viana de Souza J, Silva Dias F (2017) Protective, technological, and functional properties of select autochthonous lactic acid bacteria from goat dairy products. Curr Opin Food Sci 13:1-9. https://doi. org/10.1016/j.cofs.2017.01.003

Statistik Austria (2021) Versorgungsbilanz für Getreide 2014/15. Retrieved March 19, 2021, from http://www.statistik.at/web_de/ statistiken/wirtschaft/land_und_forstwirtschaft/preise_bilanzen/ versorgungsbilanzen/index.html

U.S. Department of Agriculture (USDA) (2020) Nutrition facts for wheat flour, soft wheat, whole-grain, recommended daily values and analysis. Retrieved March 16, 2021, from https://www.nutri tionvalue.org/Wheat_flour\%2C_soft_wheat\%2C_whole-grain nutritional_value.html

Publisher's Note Springer Nature remains neutral with regard to jurisdictional claims in published maps and institutional affiliations. 